\title{
Near-Death Experiences in Patients Undergoing Cardiopulmonary Resuscitation
}

\author{
Susan Boykoff Schoenbeck, R.N., M.S.N., C.N.S. \\ University of Wisconsin
}

Gerald D. Hocutt, M.B.A. Concordia University

ABSTRACT: The purposes of this two-phase descriptive study were to document the frequency of near-death experiences (NDEs) in a nonprobability convenience sample of patients undergoing cardiopulmonary resuscitation (CPR), to describe the types of NDE experienced most commonly during CPR, and to describe patients' views of helpful nursing responses to reports of NDEs. In Phase I a nurse read to subjects Greyson's (1983a) NDE scale. In Phase II, we used an open-ended interview designed to elicit patients' perception of helpful nursing responses to NDE reports. Results have implications for broadening the groups of persons offering patients an opportunity to discuss NDEs after CPR.

The quality of health care is not measured solely by the number of human beings who survive. Rather, it is measured by the number of patients who live on, for however long, with a sense of dignity, wellbeing, and worth. In the 1990 s we are faced with the fixed variables of

Ms. Schoenbeck is a Clinical Nurse Specialist at the University of Wisconsin Hospital and Clinics, and Mr. Hocutt is a statistical consultant and Instructor at Concordia University. This research was conducted with the support of the Veterans Administration and Beta Eta Chapter of Sigma Theta Tau, International Honor Society of Nursing. Special acknowledgement is given to Kathryn Brown, R.N., B.S.N., for data collection. Requests for reprints should be addressed to Ms. Schoenbeck at the University of Wisconsin Hospital and Clinics, Room F6/169, 600 Highland Avenue, Madison, WI 53792 . 
an increasing number of elderly patients, advancing technology, and more limited resources. We cannot afford to be fixed in our response.

On a daily basis, health care professionals face the challenge of helping their patients experience events in which human caring dominates despite the omnipresence of machinery. We try to balance the wonders of science with the needs of the human spirit. To do this, we must support a paradigm shift in which death is viewed not only as a symptom to be treated but also as a life event. Stories about people's near-death experiences (NDEs) offer us a glimpse of the process of dying. From such narratives, we can gather ideas to help those who return from death.

Albert Einstein (1930) believed that the most beautiful thing we can experience is the mysterious, which he called the source of all true art and science. The mystery of the NDE is open to those willing to believe that patients' perceptions of what happens to them are, in fact, reality. Acceptance of these data is necessary to gain knowledge of the patients' expanded level of consciousness during a near-death experience. Health professionals are continually replenished and renewed when working with the dying, who have much to teach us about living. The desired outcome of this study was to prepare health professionals better to deal with a population of patients in whom NDEs occur.

Because more people are surviving cardiopulmonary arrest, health professionals are becoming increasingly aware of patients' reports of NDEs occurring during cardiopulmonary resuscitation (CPR). NDEs have been reported in hospitalized patients who were near clinical death, in accident victims, and in suicide attempters (Bates and Stanley, 1985; Greyson, 1983a, 1983b; Noyes, 1980). Twenty-two percent of hospitalized patients who were known to be near death have reported NDEs (Bates and Stanley, 1985).

Greyson (1983a) described NDEs as comprising four componentscognitive, affective, paranormal, and transcendental-and reported (1985) that the nature of the near-death event may influence the type of NDE described.

The principal aim of this study was to document the frequency of NDEs in the population of patients who have experienced CPR. A second goal was to describe the types of NDE experienced most commonly by these patients. A third purpose was to describe patients' views of helpful nursing responses to NDE reports. We believe that understanding the extent to which patients experiencing CPR have NDEs may heighten medical personnel's attention to their patients' NDEs. Discernment of patients' perceptions of a helpful response to their NDEs may deepen and broaden the knowledge base from which 
health professionals can intervene in the event of NDEs occurring during CPR.

\section{Method}

\section{Sample}

A convenience sample was used, subjects being selected based on the following criteria: (1) each subject had experienced CPR within the past 6 months; (2) each subject understood and spoke English; and (3) each subject's physician approved the patient's participation in the study.

Eleven patients were included in the study.

\section{Procedure}

Potential subjects were identified from medical records by the head nurse and staff nurse of the coronary care unit (CCU) of a 200-bed Midwestern acute care teaching hospital, and approval was obtained from the patient's physician. Additionally, the CCU staff nurse investigator approached each potential subject, read the patient a letter describing the study, explained the purpose, method, and confidentiality of data explained, and obtained the patient's informed consent. Subjects were told they could withdraw from the study at any time without untoward consequences.

This descriptive study was carried out in two phases. In Phase I, a nurse investigator read Greyson's (1983a) 16-item NDE Scale to patients when they were in stable condition in the hospital. A copy of the patient's resuscitation flow sheet was attached to the NDE Scale so that medications the patient received and the length of CPR could be analyzed.

In Phase II, the nurse investigator conducted an open-ended interview with subjects who had NDEs as identified in Phase I. Questions asked included whether the patient thought it helps to talk about the NDE, and if so, when and with whom; and whether the patient had talked with anyone else about the NDE, and if so, when and with whom. Patients were further asked whether they would want from their nurse (a) reassurance, i.e., that others have had such experiences and that he or she is not "crazy"; (b) reassessing, i.e., help sorting out 
the experience; (c) roleplaying, i.e., practice telling others about the NDE; and (d) referral, i.e., to a specialist with whom he or she can talk about feelings.

Interviews in each phase of the study lasted 5-10 minutes.

\section{Data analysis}

For purposes of statistical analysis, the subjects were divided into two groups: those who had a positive score on the NDE Scale, indicating the presence of some NDE features; and those who had a zero score on the NDE Scale. The null hypotheses to be tested were that there are no differences between the two groups with respect to (1) age, (2) education, (3) duration of CPR, (4) time elapsed between initiation of CPR and responsiveness, and (5) time elapsed between CPR and participation in the study.

Since the alternative hypotheses state that there would be differences, two-tailed tests were used. We used the Mann-Whitney U test as the most appropriate measure to determine whether or not two small independent and randomly selected samples are from the same population with respect to some factor (Daniel, 1990; Siegel, 1956). A p value of .05 was considered significant; critical values for the statistic $U$ were taken from Robert Mason (1986).

\section{Results}

\section{Phase 1}

All eleven subjects were male. Nine were married, one was single, and one was divorced. Three of the eleven patients had experienced CPR previously.

The mean NDE Scale score of the entire sample was 2.36 (S.D. = 3.29), and the mean score of the five subjects with nonzero scores was 5.20 (S.D. $=2.95$ ). Six of the eleven subjects scored zero on the NDE Scale, indicating no features typical of NDEs. Of the remaining 5 subjects who had positive scores on the NDE Scale, indicating the presence of NDE features, two subjects scored 3 points, one scored 4 points, one scored 6 points, and one scored 10 points.

The one subject who scored 7 or higher on the NDE Scale, indicating the occurrence of a near-death experience, described an event that 
would be classified as the transcendental type by Greyson's (1985) typology. That individual reported that he saw and spoke with God in an unearthly place, where they discussed his return to earth and his quitting smoking.

The average age of the entire sample was 63.8 years (S.D. $=10.1$ ). The mean age was 69.7 years $($ S.D. $=6.7)$ for subjects without NDE features and 56.8 years (S.D. $=9.2$ ) for subjects with NDE features. This difference was significant $(U=3.0)$.

The average years of education for the entire sample was 12.0 years (S.D. = 2.7). The mean level of education was 11.0 years (S.D. $=3.3$ ) for subjects without NDE features and 13.2 years (S.D. $=1.1$ ) for subjects with NDE features. This difference was not significant $(U=8.5)$.

The average duration of CPR for the entire sample was 4.68 minutes (S.D. = 5.27). The mean duration of CPR was 5.25 minutes (S.D. = 4.94) for subjects without NDE features and 4.00 minutes (S.D. $=6.16$ ) for subjects with NDE features. This difference was not significant (U $=11.5$ ).

The mean time elapsed from initiation of CPR to responsiveness was 41.2 minutes (S.D. $=112.2$ ) for the 10 patients for whom that duration could be determined. (One subject had been pharmacologically paralyzed prior to his cardiopulmonary arrest with pancuronium bromide because he was on a mechanical ventilator, and therefore the duration of his unresponsiveness could not be ascertained.) The mean time between CPR initiation and responsiveness was 64.7 minutes (S.D. = 144.8) for subjects without NDE features. However, this mean was inordinately influenced by one subject who remained unresponsive for 360 minutes; the mean for the other five subjects without NDE features was 5.6 minutes (S.D. $=5.0$ ). The mean time between CPR and responsiveness was 6.0 minutes (S.D. $=9.4$ ) for subjects with NDE features. The difference between the two groups was not significant $(\mathrm{U}=7.5)$.

The time elapsed between CPR and participation in the study ranged from 5 to 52 days; the average for the entire sample was 17.6 days (S.D. = 16.8). The mean time between CPR and study participation was 23.0 days (S.D. $=21.5$ ) for subjects without NDE features and 11.2 days (S.D. $=5.9$ ) for subjects with NDE features. This difference was not significant $(U=11.5)$.

Two of the six subjects without NDE features had previously experienced CPR, as had one of the 5 subjects with NDE features. Of the six subjects without NDE features, one was medicated with atropine only; two received only lidocaine; one lidocaine and nitroglycerine; one lidocaine and epinephrine; and one lidocaine, atropine, and dopamine. Of 
the five subjects with NDE features, 3 were medicated only with lidocaine; one received lidocaine and atropine; and one received lidocaine, epinephrine, atropine, dopamine, and bretylium.

\section{Phase 2}

Only one subject reported an experience that scored 7 or greater on the NDE Scale. That subject stated that he was unfamiliar with NDEs prior to his experience. He viewed a helpful nursing response as one that contained the elements of (a) reassurance, e.g., statements that other people have had such experiences and that the patient was not alone nor thought to be "crazy" to have had such an experience; and (b) reassessment, e.g., statements of the nurse's willingness to listen and help the patient understand what had happened.

A second patient whose experience scored 6 points on the NDE Scale could not be interviewed because his clinical condition worsened and he expired before the initiation of Phase 2 of this study.

\section{Discussion}

Using a score of 7 or greater on the NDE Scale as the criterion for an NDE (Greyson, 1983a), only one of the eleven subjects in this study, or $9 \%$ of the sample, reported an NDE, his experience scoring 10 points on the NDE Scale. This frequency of NDEs among CPR survivors is lower than that generally reported in the literature for survivors of close brushes with death.

Several variables may have influenced this surprisingly low frequency. First, the majority of the sample was elderly, nine of the eleven subjects being over 60 years of age. Subjects who reported some NDE features were significantly younger than those who had zero scores on the NDE Scale, and the subject reporting a fullblown NDE was the second youngest in our sample, at 51 years of age.

Second, these patients were for the most part experiencing failure of several physiological systems in an acute care setting in which CPR was thrust upon them; their ability to survive CPR with the mental faculties required to describe their experiences may have been impaired. Third, patients were interviewed during the hospitalization in which their CPR occurred. Because they were still assimilating the 
experience into their lives, patients may have been reluctant to talk about an NDE. Finally, the small size of the sample raises the question of how representative it may be of the population of CPR survivors.

We found no significant difference between the two groups of subjects with respect to education, duration of CPR, elapsed time between initiation of CPR and responsiveness, or elapsed time between CPR and participation in the study. Neither did there seem to be any difference between the two groups with respect to prior CPR or medications administered during CPR.

The finding that a patient welcomes the nurse's discussion of the NDE may be extrapolated to other health care professionals. For, indeed, it appears that people experiencing an NDE turn to those who seem to understand the occurrence. Therefore, broadening the group to whom persons experiencing NDEs may turn for acceptance seems worthwhile.

The timing of discussion with patients who have experienced NDEs may also be critical. For many ill adults, management of the hour-tohour, day-to-day task of recovery is formidable. This healing takes time and energy that perhaps may not be usurped for some time by the task of integrating an experience of expanded consciousness into one's being. It may be appropriate to delay interviewing patients until they verbally offer readiness or until they are further down the road of recovery.

It has become clear from studies that health care workers do discuss with patients out-of-body and near-death experiences, and that health care workers have a great deal of knowledge about patients' perceptions of what has happened to them-in essence, their reality. Some health care personnel can recount patients' events as told to them, though they may or may not believe that patient's narrative reflects reality. In fact, there are many instances in which health care workers document a patient's experience as hallucinatory rather than as an out-of-body or near-death experience when the patient, in retrospect, was describing an NDE.

In order for patients and their families to have the support they need to integrate the NDE as a life event, we must examine the knowledge and beliefs of those personnel who encounter patients returning from death. This research may provide an impetus to explore the near-death views and experiences of health care professionals. Educational interventions can expand the knowledge, skills, and attitudes of health care workers, making their interventions more relevant to the patient's experiential needs. 


\section{References}

Bates, B., and Stanley, A. (1985). The epidemiology and differential diagnosis of neardeath experience. American Journal of Orthopsychiatry, 55, 542-549.

Daniel, W.W. (1990). Applied nonparametric statistics. Boston, MA: PWS-Kent.

Einstein, A. (1930). What I believe. Forum, October 30.

Greyson, B. (1983a). The Near-Death Experience Scale: Construction, reliability, and validity. Journal of Nervous and Mental Disease, 171, 369-375.

Greyson, B. (1983b). Near-death experiences and personal values. American Journal of Psychiatry, 140, 618-620.

Greyson, B. (1985). A typology of near-death experiences. American Journal of Psychiatry, 142, 967-969.

Mason, R.D. (1986). Statistical techniques in business and economics. Homewood, IL: Richard D. Irwin.

Noẏes, R., Jr. (1980). Attitude change following near-death experiences. Psychiatry, 43, 234-242.

Siegel, S. (1956). Nonparametric statistics for the behavioral sciences. New York, NY: McGraw-Hill. 